\title{
BREAST CRYPTOCOCCOSIS IN IMMUNOCOMPETENT PATIENTS
}

Mariana Fiori', Carlos Marino Cabral Calvano Filho, Pollyanna Dornelas Pereira', Marco Vinícius Fernandes', Daniela Omar de Souza'

${ }^{1}$ Hospital Universitário de Brasília - Brasília (DF), Brazil.

Introduction: Cryptococcosis is prevalent in immunocompromised individuals. Immunocompetent patients can develop latent infections, the breast being a rare focus of primary disease, with few reports in the literature. Case report: GHMC, female, 27 years old, married, ticketing operator, resident in Valparaíso / GO, Brazil. She denied comorbidities, use of medication, smoking or drinking, as well as contact with caves, farms, farms, wild animals and ingestion of game meat. She reported fever $\left(38^{\circ} \mathrm{C}\right)$, left mastalgia associated with hardened erythema with subsequent fistulization and removal of purulent secretion. Upon examination, she was in good general condition, with a palpable nodule of about $6 \times 4 \mathrm{~cm}$, in union of the lower quadrants (ULQ) of the left breast (LB), which was regular, soft, felt a little painful on palpation, with increased local temperature and without lymph node enlargement or papillary discharge. The ultrasound of the breasts showed a heterogeneous solid mass, with cystic areas of permeation, in ULQ of LB, of 4.2x $2.2 \mathrm{~cm}$, partially defined contours coinciding with a nodular image of $4 \mathrm{~cm}$ in the same topography in the mammography. Magnetic resonance imaging showed a nodular, irregular, hypodense image in $\mathrm{T} 1$, hyperdense in $\mathrm{T} 2$, with parietal enhancement and heterogeneous, progressive internal enhancement, in addition to capturing septa, measuring $6.1 \times 4.0 \times 4.6 \mathrm{~cm}$, suggesting mucinous carcinoma. Core biopsy of the solid part of the lesion and collection of mucinous fluid was performed. Concomitantly, oxacillin was started for seven days. There was no laboratory change during the entire disease period. Fifteen days after the end of the antibiotic use, the lesion became an erythematous lenticular ulcer, with flat edges, of 5.0x4.0 cm, with colloid secretion leaving its bed. Histology showed cryptococcosis, and liquid cytology showed cryptococcus neoformans. During immunosuppression investigation, the patient underwent chest and skull CT scans, serology, tumor markers, ANF (antinuclear factor), rheumatoid factor, C3, C4, lumbar puncture and blood cultures (all excluded any immunosuppressive pathology). The treatment was carried out with Fluconazole $800 \mathrm{mg} /$ day for three months, with a reduction to $300 \mathrm{mg} /$ day for another three months. Two months after the start of treatment, the lesion resolved. Cryptococcosis is an invasive mycosis with high morbidity and mortality. It affects immunosuppressed individuals, and is rare in immunocompetent individuals. The main pathogenic species, C. neoformans and C. gatti, are prevalent in tropical and subtropical climates. The main sites affected are the brain and the lungs; other sites are rare. The dosage and duration of breast therapy is unknown, but 2-3g/day of amphotericin B or 400-800 mg/day of fluconazole for 8-12 weeks has achieved therapeutic success in reported cases. 